\title{
Le VIH au Canada - Rapport de surveillance, 2018
}

\author{
N Haddad ${ }^{1}$, A Robert ${ }^{1}$, A Weeks ${ }^{1}$, N Popovic ${ }^{1}$, W Siu ${ }^{1}$, C Archibald ${ }^{1}$
}

\section{Résumé}

Contexte : Le virus de l'immunodéficience humaine $(\mathrm{VIH})$ est un problème de santé publique mondial. Le nombre de personnes vivant avec le VIH en 2017 est estimé à 36,9 millions. Le VIH doit être déclaré au Canada depuis 1985 et l'Agence de la santé publique du Canada (ASPC) continue de surveiller la tendance des nouveaux diagnostics de $\mathrm{VIH}$.

Objectif : Ce rapport de surveillance vise à donner un aperçu global de l'épidémiologie de tous les diagnostics déclarés de VIH au Canada depuis 1985, ainsi que par lieu géographique, groupe d'âge, sexe et catégorie d'exposition, tout en mettant l'accent sur 2018.

Méthodes : L'ASPC surveille le VIH au moyen du système national de surveillance du VIH/ sida, un système passif fondé sur les cas, qui recueille des données non nominatives soumises volontairement par toutes les provinces et tous les territoires canadiens. Des analyses épidémiologiques descriptives ont été effectuées sur les données nationales et celles portant sur des populations spécifiques fournies par Immigration, Réfugiés et Citoyenneté Canada et le Programme de surveillance périnatale du VIH au Canada.

Résultats : En 2018, un total de 2561 diagnostics de VIH ont été signalés au Canada, soit une augmentation de 8,2 \% par rapport à 2017. Le taux national de diagnostic est passé de 6,5 pour 100000 habitants en 2017 à 6,9 pour 100000 habitants en 2018. La Saskatchewan a enregistré le taux de diagnostic provincial le plus élevé, soit 14,9 pour 100000 habitants. Le groupe des 30 à 39 ans a continué d'afficher le taux de diagnostic du VIH le plus élevé, soit 15,4 pour 100000 habitants. Dans l'ensemble, le taux de diagnostic chez les hommes a continué d'être plus élevé que chez les femmes $(9,8$ contre 4,0 pour 100000 habitants, respectivement); toutefois, le nombre de cas déclarés et le taux de diagnostics chez les femmes ont connu une augmentation plus importante. La catégorie d'exposition des hommes gais, bisexuels et autres hommes ayant des rapports sexuels avec des hommes (gbHARSAH) a continué de représenter la plus forte proportion de tous les cas déclarés chez les adultes $(41,4 \%)$, bien que cette proportion ait diminué avec le temps. Cinq cas de transmission du VIH durant la période périnatale ont été documentés; dont trois cas où la mère n'a pas reçu de traitement antirétroviral périnatal prophylactique.

Conclusion : Le nombre et le taux de cas déclarés de VIH au Canada ont augmenté en 2018. Les hommes gbHARSAH demeure la catégorie d'exposition la plus fréquente, tandis que le nombre et le taux de cas déclarés de VIH chez les femmes ont augmenté. L'ASPC continuera de collaborer avec ses partenaires nationaux pour améliorer la collecte, l'analyse et la publication de données nationales afin de mieux comprendre le fardeau du VIH au Canada.

Citation proposée : Haddad N, Robert A, Weeks A, Popovic N, Siu W, Archibald C. Le VIH au Canada - Rapport de surveillance, 2018. Relevé des maladies transmissibles au Canada 2019;45(12):334-43.

https://doi.org/10.14745/ccdr.v45i12a01f

Mots-clés : VIH, surveillance, hommes gais, bisexuels et autres hommes ayant des rapports sexuels avec des hommes, utilisateurs de drogues par injection, VIH périnatal, contact hétérosexuel, Canada
Cette oeuvre est mise à la disposition selon les termes de la licence internationale Creative Commons Attribution 4.0

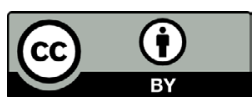

Affiliations

${ }^{1}$ Centre de la lutte contre les maladies transmissibles et les infections, Agence de la santé publique du Canada, Ottawa, ON

*Correspondance :

phac.hass.aspc@canada.ca 


\section{Introduction}

Le virus de l'immunodéficience humaine $(\mathrm{VIH})$ demeure un problème de santé publique mondial. En 2017, le nombre estimé de personnes vivant avec le VIH était passé à 36,9 millions (1). Le VIH doit être déclaré au Canada depuis 1985 et l'Agence de la santé publique du Canada (ASPC) continue de surveiller la tendance des nouveaux diagnostics de VIH. Au Canada, en 2017, 2402 nouveaux diagnostics de VIH ont été déclarés avec un taux de diagnostic de 6,5 pour 100000 habitants (2), ce qui représente une augmentation par rapport à 2014 où le taux de nouveaux diagnostics de VIH était de 5,8 pour 100000 habitants (2). Par ailleurs, environ 686,8 millions de dollars ont été consacrés à la prévention et au traitement du VIH au Canada en 2015 , ce qui démontre l'importance du fardeau économique de la maladie (3).

Récemment, I'ASPC a publié un cadre d'action pancanadien et un plan d'action quinquennal connexe sur les infections transmissibles sexuellement ou par le sang, qui décrivent en détail l'importance d'une approche commune pour traiter les populations clés touchées de façon disproportionnée par ces infections $(4,5)$. Le diagnostic rapide et la déclaration du VIH sont essentiels pour surveiller les tendances des infections nouvellement diagnostiquées afin d'informer et d'évaluer les programmes de prévention et de soins (6-9).

Ce rapport de surveillance vise à donner un aperçu global de l'épidémiologie de tous les diagnostics déclarés de $\mathrm{VIH}$ au Canada depuis 1985 jusqu'à la fin de 2018, ainsi que par lieu géographique, groupe d'âge, sexe et catégorie d'exposition. Comme dans les rapports précédents, nous présentons ici des renseignements à jour sur les résultats du dépistage médical du VIH aux fins d'immigration et sur le nombre de nourrissons exposés au VIH et infectés par le $\mathrm{VIH}$ pendant la période périnatale. Le présent rapport traite également des travaux récents de I'ASPC visant à quantifier et à expliquer l'effet des cas de VIH déjà diagnostiqués sur la tendance des nouveaux diagnostics de VIH au Canada (10). De plus, bien que I'ASPC ait publié par le passé des rapports de surveillance concernant les cas de sida nouvellement diagnostiqués, l'Agence déclare les cas de sida de 2018 au moyen du système canadien de surveillance des maladies à déclaration obligatoire (11) et, donc, les cas de sida ne seront pas présentés ici.

Il est important de savoir que les données de surveillance ne décrivent que la partie diagnostiquée de l'épidémie de VIH. Les estimations nationales de la prévalence et de l'incidence du $\mathrm{VIH}$ qui décrivent l'épidémie du $\mathrm{VIH}$ en général au Canada, y compris les personnes ayant reçu ou non un diagnostic de $\mathrm{VIH}$, sont produites à l'aide de modèles statistiques et de sources d'information supplémentaires qui sont publiés séparément (12).

\section{Méthodes}

\section{Sources de données}

Les données présentées dans ce rapport de surveillance du VIH proviennent de trois sources différentes : le Système national de surveillance du VIH/sida (SSVS) de I'ASPC, le Dépistage médical du VIH aux fins d'immigration provenant d'Immigration, Réfugiés et Citoyenneté Canada (IRCC) et le Programme de surveillance périnatale du VIH au Canada (PSPVC).

\section{Système national de surveillance du $\mathrm{VIH} /$ sida}

Le Système national de surveillance du $\mathrm{VIH} /$ sida est un système de surveillance passif, basé sur les cas, qui recueille des données non nominales sur les personnes séropositives dont le cas respecte la définition nationale de cas (13). Les autorités provinciales et territoriales de la santé publique fournissent volontairement à l'ASPC leurs données, notamment l'âge, le sexe, la race ou l'origine ethnique et les risques associés à la transmission du VIH (catégories d'exposition). Aux fins de ce rapport, un " adulte » est une personne de 15 ans ou plus.

Il est important de noter que le Québec ne transmet pas à I'ASPC des renseignements sur la catégorie d'exposition ou la race ou l'origine ethnique pour les cas de $\mathrm{VIH}$, alors que la Colombie-Britannique ne transmet pas à l'ASPC des renseignements sur la race ou l'origine ethnique pour les cas de $\mathrm{VIH}$ et l'Ontario ne dispose pas de données sur la race ou l'origine ethnique des cas déclarés avant 2009. De plus, en ce qui concerne la race ou l'origine ethnique, en 2018 la Saskatchewan a classé les cas sous Autochtones ou non-Autochtones uniquement, sans autre ventilation.

Les provinces et les territoires fournissent des données au moyen du formulaire de déclaration de cas (14) à l'échelle nationale par la transmission sécurisée d'un ensemble de données électroniques. Toutes les données brutes (formulaires papier et ensembles de données électroniques) sont conservées en conformité avec la Directive sur la collecte, I'utilisation et la diffusion de l'information sur la santé publique (ASPC, 2013, document non publié). L'évaluation de la qualité des données, comme la détection des entrées en double, est réalisée par les provinces et les territoires qui les soumettent par la suite à I'ASPC. Les données présentées dans ce rapport de surveillance représentent les nouveaux cas de $\mathrm{VIH}$ diagnostiqués jusqu'au 31 décembre 2018 qui ont été présentés à l'ASPC par les programmes de surveillance provinciaux et territoriaux jusqu'au 12 juillet 2019. Vous trouverez ailleurs d'autres détails sur les méthodes du système national de surveillance du VIH/sida $(2,14)$.

\section{Dépistage médical du VIH aux fins d'immigration}

Tous les ressortissants étrangers de 15 ans et plus qui présentent une demande de résidence permanente au Canada et certains de ceux qui présentent une demande de résidence temporaire 
doivent subir un examen médical aux fins de l'immigration (EMI) que leur fait passer IRCC, soit au Canada ou à l'étranger. Ce ministère fournit par la suite à l'ASPC des données non nominales recueillies durant l'EMI sur les migrants qui étaient séropositifs au VIH, soit au Canada ou à l'étranger. Le terme migrant est utilisé au sens large et inclut notamment les immigrants (les résidents permanents du Canada), les réfugiés, les demandeurs d'asile ou les réfugiés au sens de la Convention et les résidents temporaires (visiteurs, étudiants ou travailleurs étrangers). Les données d'EMI présentées ici proviennent du Système mondial de gestion des cas d'IRCC, mis à jour jusqu'en mars 2019, et qui contient l'information d'EMI pour tous les candidats sélectionnés au Canada ou à l'étranger qui étaient séropositifs au VIH. Les données agrégées ont été fournies à I'ASPC en juillet 2019. Les données présentées dans ce rapport portent sur les personnes qui ont été testées au Canada.

IRCC partage avec les provinces et les territoires les données nominales des résultats des EMI effectués à l'étranger pour tous les clients qui ont reçu un diagnostic de $\mathrm{VIH}$ et qui ont une adresse résidentielle canadienne valide dans leur dossier qui indique leur province ou leur territoire de résidence actuel. Ces données peuvent par la suite être intégrées, à différents degrés, aux systèmes de surveillance provinciaux et territoriaux systématiques du VIH basés sur les cas; certains territoires et provinces considèrent ces cas de migrants séropositifs comme de nouveaux diagnostics alors que d'autres les excluent de la déclaration provinciale ou territoriale à I'ASPC.

\section{Programme de surveillance périnatale du VIH au Canada}

Les données nationales sur la séropositivité des nourrissons exposés au VIH durant la période périnatale sont recueillies par le PSPVC, une initiative du Groupe canadien de recherche sur le sida chez les enfants. Le PSPVC est un système de surveillance sentinelle actif qui recueille des données sur deux groupes d'enfants : les nourrissons nés de femmes séropositives et les enfants séropositifs au VIH qui reçoivent des soins dans des sites participants (qu'ils soient nés au Canada ou à l'étranger). Des renseignements supplémentaires sur la méthodologie du PSPVC ont été décrits précédemment (2). Les données de surveillance pour 2018, y compris les mises à jour des données pour les années précédentes, ont été soumises à l'ASPC en mars 2019.

\section{Analyse}

Les tendances descriptives globales et par région géographique, groupe d'âge, sexe, catégorie d'exposition et population clé sont présentées. Les logiciels Excel 2010 de Microsoft (Redmond, État de Washington, États-Unis) et SAS Enterprise Guide v7.1 (Cary, Caroline du Nord, États-Unis) ont servi au nettoyage et à l'analyse des données. On a appliqué des procédures normalisées de recodage des données à tous les ensembles de données provinciales et territoriales soumises pour créer un ensemble de données nationales aux fins d'analyse. Les données de surveillance présentées dans ce rapport ont été validées par toutes les provinces et tous les territoires pour en assurer l'exactitude. Aucune procédure statistique n'a été utilisée pour l'analyse comparative et l'on n'a appliqué aucune technique statistique pour tenir compte des données manquantes puisque les analyses étaient limitées aux tableaux croisés en raison de la nature descriptive de l'analyse. Les proportions présentées dans le texte excluent les dossiers présentant des valeurs manquantes (sauf indication contraire).

Les Statistiques démographiques annuelles, publiées par Statistique Canada en juillet 2018 (15), constituaient la source de données sur la population utilisée pour calculer les taux.

Les tableaux supplémentaires sont inscrits dans l'annexe et sont disponibles sur demande.

\section{Résultats}

\section{Tendances générales}

Au total, 88881 diagnostics de VIH ont été signalés à l'ASPC depuis le début de la déclaration du VIH au Canada en 1985. En 2018, un total de 2561 diagnostics de VIH ont été signalés, soit une augmentation de 8,2 \% par rapport à 2017. Le taux national de diagnostic a fluctué au fil des ans avec une tendance à la baisse entre 2008 et 2015, suivie d'une légère augmentation à 6,9 pour 100000 habitants en 2018 (figure 1).

Figure 1 : Nombre de cas signalés de VIH, et les taux de diagnostic globaux, selon le sexe et l'année, Canada, de 1996 à $2018^{a}$

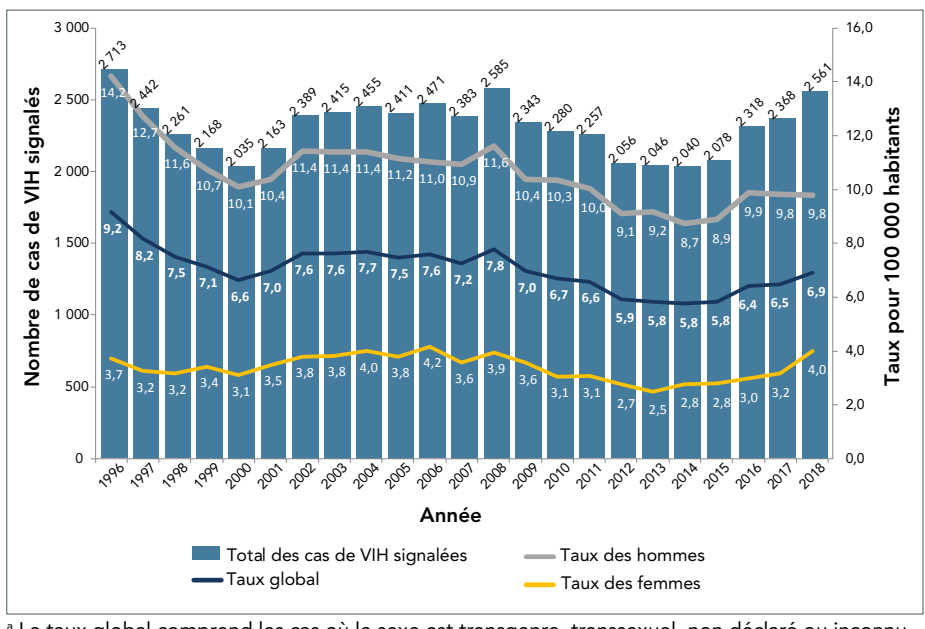

a Le taux global comprend les cas où le sexe est transgenre, transsexuel, non déclaré ou inconnu

Depuis 2009, les taux de diagnostic chez les hommes et les femmes ont fluctué. Chez les hommes, il y a eu une légère baisse depuis 2009 (10,4 pour 100000 habitants en 2009, à 9,8 pour 100000 habitants en 2018). Chez les femmes, le taux de diagnostics de VIH a fluctué entre 2,5 et 4,2 pour 100000 
habitants depuis 1996. Au cours des cinq dernières années, le taux est passé de 2,5 pour 100000 habitants en 2013 à 3,2 pour 100000 habitants en 2017 et 4,0 pour 100000 habitants en 2018.

\section{Distribution géographique}

En 2018, I'Ontario a affiché le nombre le plus élevé et la proportion la plus élevée de cas de VIH signalés ( $n=1003$, $39,2 \%$ ), suivi du Québec ( $n=766,29,9 \%$ ), de l'Alberta ( $n=249,9,7 \%$ ) et de la Colombie-Britannique ( $n=199,7,8 \%$ ). Comme on l'a vu en 2017, les taux provinciaux et territoriaux de diagnostics de $\mathrm{VIH}$ déclarés variaient d'une région à l'autre du Canada. La Saskatchewan a continué d'afficher le taux de diagnostic le plus élevé, soit 14,9 pour 100000 habitants, bien que la province ne représente que 6,8 \% du nombre total de cas de $\mathrm{VIH}$ déclarés. Le Québec vient au deuxième rang avec 9,1 pour 100000 habitants, suivi du Manitoba (7,9 pour 100000 habitants) et de l'Ontario (7,0 pour 100000 habitants) (figure 2). Des augmentations du taux de diagnostic du VIH ont été observées au Québec, au Manitoba et en Ontario (de 8,1, 6,7 et 6,5 pour 100000 habitants en 2017, respectivement), tandis que la Saskatchewan et l'Alberta ont connu des baisses (de 15,6 et 6,8 pour 100000 habitants en 2017, respectivement). On a observé une augmentation des taux de diagnostic chez les femmes en Colombie-Britannique, en Saskatchewan, au Manitoba, en Ontario et au Québec en 2018 par rapport à 2017.

Figure 2 : Taux de diagnostic du VIH (pour 100000 habitants) par province et territoire, Canada, $2018^{\circ}$

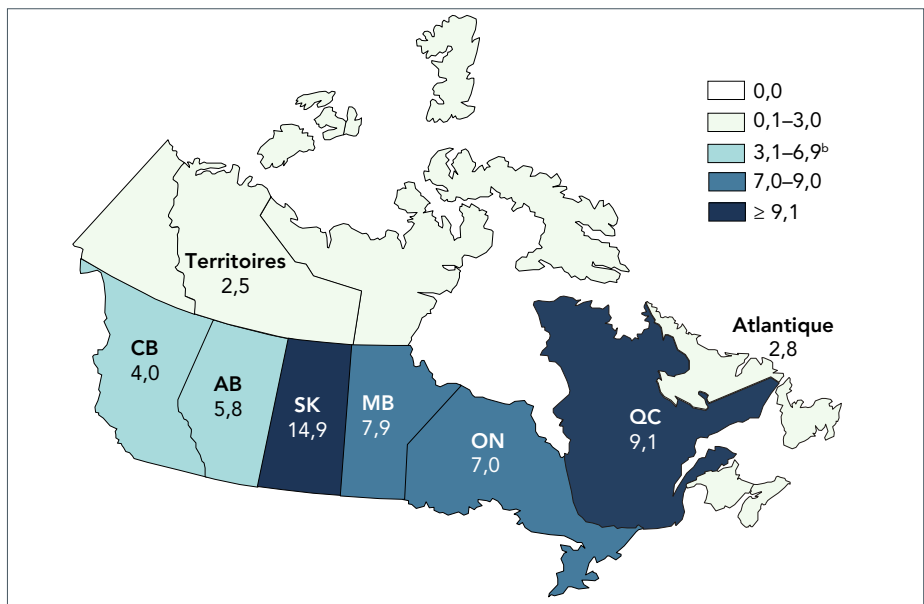

Abréviations: $A B$, Alberta; $C B$, Colombie-Britannique; $M B$, Manitoba; ON, Ontario; $\mathrm{OC}$, Québec; $\mathrm{SK}$, Saskatchewan; $\geq$, supérieur ou égal à

Les taux pour les territoires (Yukon, Nunavut et Territoires du Nord-Ouest) et la région de I'Atlantique (Île-du-Prince-Édouard, Nouveau-Brunswick, Nouvelle-Écosse et Terre-Neuve-et-

Labrador) sont présentés en moyennes

Taux national de 6,9 cas pour 100000 habitants

\section{Répartition par groupe d'âge et par sexe}

En 2018, les données sur les groupes d'âge étaient disponibles pour près de $100 \%(n=2558)$ de tous les cas de VIH déclarés. Le groupe des 30 à 39 ans continue d'avoir le plus grand nombre et la plus forte proportion de cas déclarés de $\mathrm{VIH}(n=778$, $30,4 \%$, ce qui est semblable à 2017. Les groupes d'âge de
50 ans et plus et de 20 à 29 ans ont présenté la deuxième plus forte proportion de cas de VIH déclarés, soit $22,5 \%$ pour chaque groupe d'âge ( $n=576$ et $n=575$, respectivement). Vient ensuite le groupe de 40 à 49 ans ( $n=559,21,9 \%$ ). Le groupe des 15 à 19 ans et les enfants (moins de 15 ans) représentaient respectivement $1,6 \%$ et $1,1 \%$ des nouveaux diagnostics de $\mathrm{VIH}$.

Par conséquent, le groupe des 30 à 39 ans présentait le taux le plus élevé de cas de VIH déclarés, soit 15,4 pour 100000 habitants (figure 3). Le groupe des 40 à 49 ans vient au deuxième rang avec 11,7 pour 100000 habitants, suivi du groupe des 20 à 29 ans avec 11,5 pour 100000 habitants. Le groupe des 20 à 29 ans et celui des 40 à 49 ans ont des taux similaires depuis 2016. Dans l'ensemble, les taux de diagnostic chez les enfants (moins de 15 ans), les 15 à 19 ans et les 50 ans et plus sont demeurés relativement stables au cours des cinq dernières années.

Figure 3 : Taux de diagnostics de $\mathrm{VIH}$, par groupe d'âge et année, Canada, de 2014 à 2018

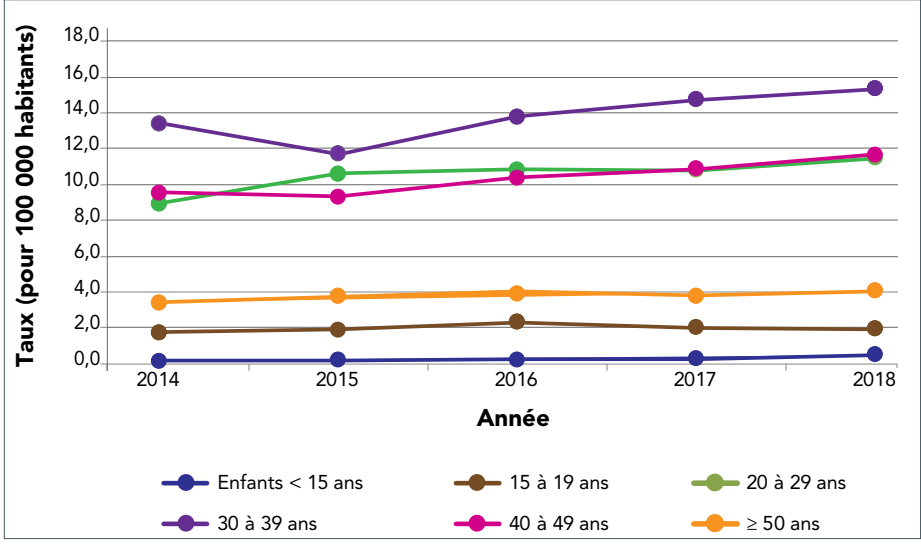

Abréviations : $<$ moins de $>$ égal ou plus de

axclut les cas pour lesquels l'âge n'est pas déclaré ou connu

Source de données démographiques: Statistiques démographiques annuelles, Statistique Canada (15)

En 2018, les données sur le sexe étaient disponibles pour près de $100 \%$ ( $n=2552)$ de tous les cas de VIH déclarés. Les hommes représentaient $70,7 \%$ tandis que les femmes représentaient $29,3 \%$. II s'agit d'un léger changement dans la proportion entre les sexes par rapport à 2017, où les hommes représentaient $75,3 \%$ et les femmes $24,7 \%$ pour les cas déclarés de VIH dont le sexe était connu. Le groupe des 30 à 39 ans présentait la plus forte proportion de cas de $\mathrm{VIH}$ chez les hommes ( $n=521$, $28,9 \%$ ) comme chez les femmes ( $n=256,34,2 \%$ ) (figure 4). Les proportions de cas de $\mathrm{VIH}$ dans les groupes des 40 à 49 ans et des 50 ans et plus étaient similaires pour les hommes et les femmes. Cependant, la proportion de cas de VIH était plus faible chez les femmes de 20 à 29 ans et plus élevée chez les 15 à 19 ans et les moins de 15 ans.

À l'instar de la tendance générale, les taux de diagnostic les plus élevés ont été observés dans le groupe des 30 à 39 ans, soit 20,5 pour 100000 habitants chez les hommes et 10,1 pour 100000 
Figure 4 : Proportion de nouveaux cas de $\mathrm{VIH}$, par groupe d'âge et sexe, Canada, 2018 a,b

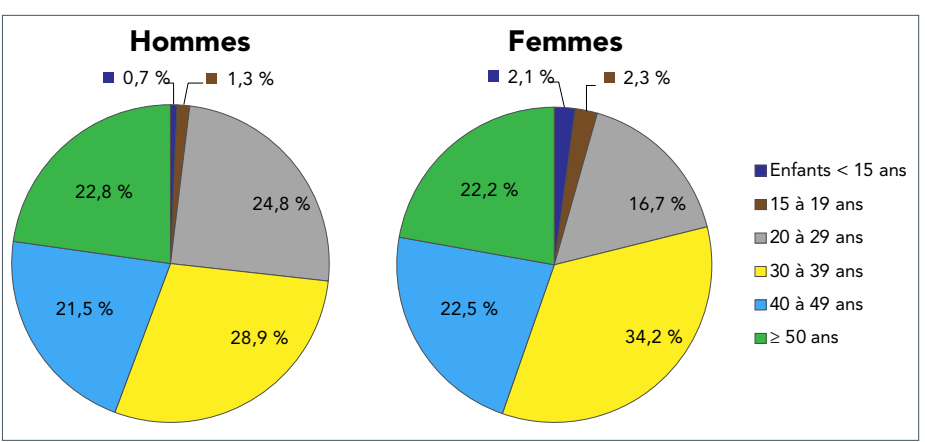

Abréviations : $<$, moins de; $\geq$, égal ou plus de

a Exclut les cas pour lesquels le sexe est transsexuel, transgenre, non déclaré ou inconnu

${ }^{b}$ Exclut les cas pour lesquels l'âge n'est pas déclaré ou connu

habitants chez les femmes. Si l'on observe les taux dans les autres groupes d'âge, les groupes des 20 à 29 ans et des 40 à 49 ans affichaient les deuxième et troisième taux de diagnostic les plus élevés chez les hommes, soit 17,2 pour 100000 habitants et 16,4 pour 100000 habitants, respectivement. La tendance inverse a été observée chez les femmes, où le groupe des 40 à 49 ans avait le deuxième taux de diagnostic le plus élevé, soit 7,0 pour 100000 habitants, suivi du groupe des 20 à 29 ans avec un taux de 5,2 pour 100000 habitants.

Dans l'ensemble, depuis 2014, les hommes de 20 à 29 ans ont connu la plus forte augmentation des taux de diagnostic du VIH, tandis que la plus forte augmentation des taux de diagnostic du VIH a été enregistrée chez le groupe de femmes de 30 à 39 ans, suivi par le groupe de 40 à 49 ans.

\section{Répartition des catégories d'exposition}

Les tendances dans les catégories d'exposition ont changé depuis le début de la déclaration du VIH en 1985. Au fil des ans, bien que la catégorie d'exposition des hommes gais, bisexuels et autres hommes ayant des rapports sexuels avec des hommes (gbHARSAH) ait continué de représenter la plus forte proportion de cas, cette proportion a diminué. En 2018, parmi tous les cas pour lesquels la catégorie d'exposition était connue $(n=1462$, $57,1 \%), 41,4 \%$ de tous les cas déclarés chez les adultes $(n=600$ sur 1450 ) ont été attribués à la catégorie d'exposition des gbHARSAH (tableau 1). Cela représentait une baisse par rapport aux $46.6 \%$ de 2017. La deuxième catégorie d'exposition la plus fréquente chez les adultes a continué d'être le contact hétérosexuel, soit $32,3 \%$ des cas d'exposition connus chez les adultes. Un changement a été observé dans la distribution des proportions pour chaque sous-groupe de contact hétérosexuel au cours des cinq dernières années. La proportion des trois sous-groupes hétérosexuels était presque égale en 2014 , avec une moyenne de $9,6 \%$, alors qu'en 2018 , cette répartition était comme suit :15,4\% attribué aux contacts hétérosexuels entre personnes nées dans un pays où le $\mathrm{VIH}$ est endémique (Hét-Endémique), 6,2 \% attribué aux contacts hétérosexuels avec une personne à risque (Hét-Risque) et 10,7\% attribué
Tableau 1 : Nombre et proportion de cas de VIH par sexe et catégorie d'exposition chez les adultes âgés de 15 ans ou plus, Canada (sauf le Québec), 2017 à $2018^{\text {a-e }}$

\begin{tabular}{|c|c|c|c|c|c|c|c|c|c|c|c|c|}
\hline \multirow{3}{*}{$\begin{array}{c}\text { Catégorie } \\
\text { d'exposition }\end{array}$} & \multicolumn{6}{|c|}{2017} & \multicolumn{6}{|c|}{2018} \\
\hline & \multicolumn{2}{|c|}{ Homme } & \multicolumn{2}{|c|}{ Femmes } & \multicolumn{2}{|c|}{ Total $^{a}$} & \multicolumn{2}{|c|}{ Homme } & \multicolumn{2}{|c|}{ Femmes } & \multicolumn{2}{|c|}{ Total ${ }^{a}$} \\
\hline & $\mathrm{n}$ & $\%$ & $n$ & $\%$ & $n$ & $\%$ & $n$ & $\%$ & $\mathrm{n}$ & $\%$ & $\mathrm{n}$ & $\%$ \\
\hline gbHARSAH & 667 & 61,0 & $n / a$ & $n / a$ & 667 & 46,6 & 600 & 58,1 & $\mathrm{n} / \mathrm{a}$ & $n / a$ & 600 & 41,4 \\
\hline gbHARSAH/UDI & 39 & 3,6 & $n / a$ & $n / a$ & 39 & 2,7 & 49 & 4,7 & $\mathrm{n} / \mathrm{a}$ & $n / a$ & 49 & 3,4 \\
\hline UDI & 138 & 12,6 & 93 & 27,6 & 232 & 16,2 & 146 & 14,1 & 118 & 28,4 & 265 & 18,3 \\
\hline $\begin{array}{l}\text { Contact } \\
\text { hétérosexuel }\end{array}$ & 204 & 18,6 & 207 & 61,4 & 412 & 28,8 & 200 & 19,4 & 266 & 64,1 & 468 & 32,3 \\
\hline $\begin{array}{l}\text { a) originaire d'un } \\
\text { pays où le VIH est } \\
\text { endémique }\end{array}$ & 62 & 5,7 & 105 & 31,2 & 167 & 11,7 & 82 & 7,9 & 140 & 33,7 & 223 & 15,4 \\
\hline $\begin{array}{l}\text { b) contact } \\
\text { sexuel avec une } \\
\text { personne à risque }\end{array}$ & 51 & 4,7 & 46 & 13,6 & 98 & 6,8 & 37 & 3,6 & 52 & 12,5 & 90 & 6,2 \\
\hline $\begin{array}{l}\text { c) aucun risque } \\
\text { relevé }\end{array}$ & 91 & 8,3 & 56 & 16,6 & 147 & 10,3 & 81 & 7,8 & 74 & 17,8 & 155 & 10,7 \\
\hline Autre $^{b}$ & 46 & 4,2 & 37 & 11,0 & 83 & 5,8 & 37 & 3,6 & 31 & 7,5 & 68 & 4,7 \\
\hline Total partiel $^{\mid}$ & 1094 & 100,0 & 337 & 100,0 & 1433 & 100,0 & 1032 & 100,0 & 415 & 100,0 & 1450 & 100,0 \\
\hline $\begin{array}{l}\text { Aucun risque } \\
\text { relevéd }^{d}\end{array}$ & 60 & 3,4 & 8 & 1,4 & 69 & 2,9 & 54 & 3,0 & 22 & 3,0 & 77 & 3,0 \\
\hline $\begin{array}{l}\text { Catégorie } \\
\text { d'exposition } \\
\text { inconnue ou non } \\
\text { signalée } \\
\text { (" manquante ») }\end{array}$ & 613 & 34,7 & 230 & 40,0 & 846 & 36,0 & 703 & 39,3 & 295 & 40,3 & 1002 & 39,6 \\
\hline Total & 1767 & $n / a$ & 575 & $\mathrm{n} / \mathrm{a}$ & 2348 & $n / a$ & 1789 & $n / a$ & 732 & $n / a$ & 2529 & $\mathrm{n} / \mathrm{a}$ \\
\hline
\end{tabular}

Abréviations : gbHARSAH, gais, bisexuel et autres hommes ayant des rapports sexuels avec des hommes; S.O., sans objet; UDI, utilisateurs de drogues par injection

a Le total des colonnes inclut les cas de personnes transsexuelles ou transgenres, ainsi que les cas pour lesquels le sexe n'a pas été signalé, alors que les colonnes « Hommes » et "Femmes " excluent ces cas

b "Autre » inclut les cas de l'Alberta relevés par Immigration, Réfugiés et Citoyenneté Canada, les cas de coagulation du sang et d'autres catégories d'exposition

c Les proportions sont basées sur le total partiel de la catégorie d'exposition connue

${ }^{d}$ Aucun risque relevé : Utilisé lorsque les antécédents d'exposition au VIH par l'un ou l'autre des autres modes énumérés sont inconnus, ou lorsqu'aucun antécédent d'exposition n'a été signalé (p. ex. décès ou manque de suivi) (14)

- Inclut tous les cas pour lesquels la catégorie d'exposition n'était pas connue ni signalée. Comme le Québec n'a pas fourni d'information sur les catégories d'exposition, les nouveaux diagnostics de VIH déclarés par le Québec sont inclus ici

aux contacts hétérosexuels sans risque identifié (Hét-ARR). Les utilisateurs de drogues par injection (UDI) représentaient le troisième plus grand nombre de cas de $\mathrm{VIH}$ parmi les adultes en 2018 , soit $18,3 \%$ (tableau 1 ).

L'analyse de la variable de la catégorie d'exposition a été effectuée séparément pour les hommes et les femmes puisque la catégorie d'exposition des gbHARSAH s'applique uniquement aux hommes. Chez les hommes adultes en 2018, la catégorie d'exposition des gbHARSAH représentait la plus forte proportion $(58,1 \%)$ des cas déclarés, et chez les femmes adultes, l'exposition par contact hétérosexuel représentait la plus forte proportion, soit $64,1 \%(33,7$ \% Hét-Endémique, 12,5 \% HétRisque et $17,8 \%$ Hét-ARR). En outre, chez les femmes adultes, les UDI représentaient un peu plus du quart des cas de $\mathrm{VIH}$ $(28,4 \%)$ comparativement aux $14,1 \%$ chez les hommes adultes (tableau 1).

En 2018, plus d'un tiers des cas de VIH déclarés chez les gbHARSAH étaient âgés de 20 à 29 ans $(35,5 \%)$ et environ un tiers étaient âgés de 30 à 39 ans (32\%) (figure 5). De même, $30,6 \%$ des cas de VIH chez les gbHARSAH/UDI étaient âgés de 20 à 29 ans, et une distribution similaire a été observée pour les cas âgés de 30 à 39 ans, de 40 à 49 ans et ceux de plus 
Figure 5 : Proportion des cas déclarés de VIH (âgés de 15 ans ou plus) par catégorie d'exposition et groupe d'âge, Canada, 2018 a,b

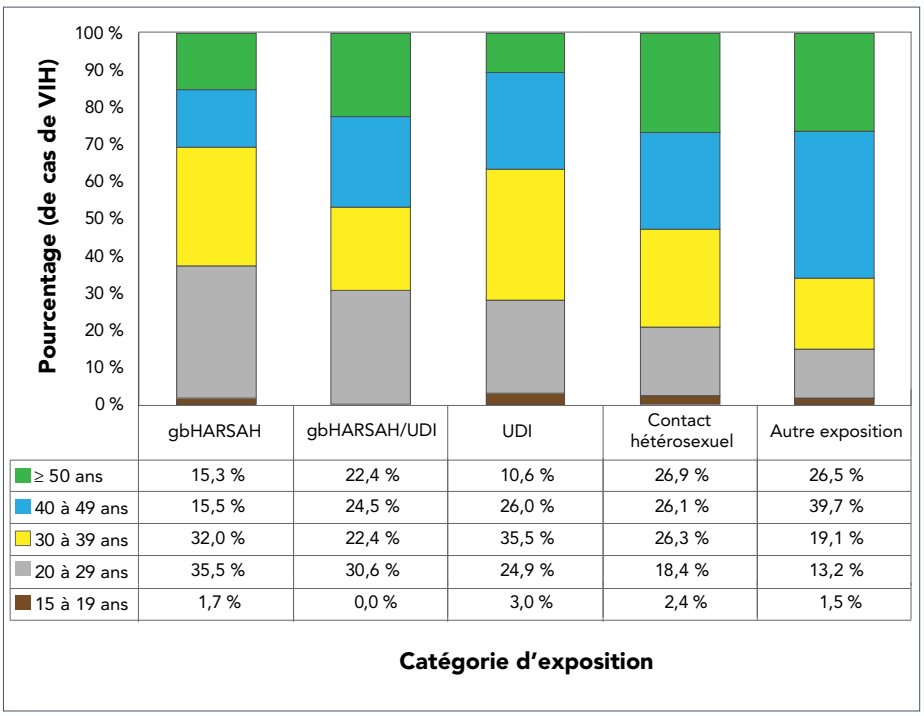

Abréviations : gbHARSAH; gais, bisexuels et autres hommes ayant des rapports sexuels avec des hommes; UDI, utilisateurs de drogues par injection; $\geq$, égal ou plus de

a Exclut les cas pour lesquels la catégorie d'exposition n'a pas été signalée

'La catégorie "Autre exposition » comprend les cas de l'Alberta relevés par Immigration, Réfugiés et Citoyenneté Canada, les cas de coagulation du sang et d'autres catégories d'exposition

de 50 ans ou plus $(22,4 \%, 24,5 \%$ et $22,4 \%$, respectivement). Dans le groupe à risque des UDI, des cas ont été signalés dans un groupe légèrement plus âgé que les gbHARSAH et le gbHARSAH/UDI avec 35,5\% des cas âgés de 30 à 39 ans. Les cas signalés dans la catégorie d'exposition par contact hétérosexuel étaient également répartis entre les groupes d'âge de 30 à 39 ans, de 40 à 49 ans et plus de 50 ans ou plus $(26,3 \%$, $26,1 \%$ et $26,9 \%$, respectivement).

\section{Renseignements sur la race ou l'origine ethnique}

Au fil des ans, les données nationales recueillies sur la race ou l'origine ethnique n'ont pas toujours été complètes. Ces données ne sont pas présentées dans ce rapport, comme elles l'ont été dans les rapports précédents, pour deux raisons principales. Premièrement, les renseignements sur la race ou l'origine ethnique étaient disponibles pour moins de la moitié des cas déclarés de VIH en 2018 ( $n=1$ 196, 46,7 \%, sans compter le Québec et la Colombie-Britannique). De plus, en 2018, pour la première fois, la Saskatchewan a attribué les cas à deux catégories seulement : autochtone et non autochtone, sans autre précision ou catégorie. Deuxièmement, en raison de l'évolution de la population diversifiée du Canada et des récents changements apportés à la méthodologie de Statistique Canada pour catégoriser l'origine ethnique (16), il faudra peut-être réévaluer les catégories actuelles pour la race ou l'origine ethnique.

Parmi les cas pour lesquels des renseignements sur la race ou l'origine ethnique étaient connus, $19,3 \%$ ont été signalés comme autochtones, et $80,7 \%$ comme autres races ou origines ethniques. Ces résultats sont semblables à ceux observés en 2017, alors que 20,1\% étaient considérés comme autochtones et $79,9 \%$ comme d'autres races ou ethnies.

\section{Dépistage médical du VIH aux fins d'immigration}

En 2018, un total de 1026 migrants se sont révélés séropositifs au VIH durant I'EMI. De ce nombre, 696 ont été testés au Canada (figure 6) et le reste à l'étranger. Cela représente une augmentation par rapport à 2017, année où un total de 835 migrants se sont révélés séropositifs durant l'EMI, dont 549 au Canada. Les migrants qui se sont révélés séropositifs durant I'EMI au Canada sont mis en évidence dans le présent rapport parce que ces personnes sont testées dans les systèmes de santé publique provinciaux et territoriaux et sont donc incluses dans le Système national de surveillance du VIH/sida (SSVS).

Figure $6:$ Nombre de migrants qui se sont révélés séropositifs durant un examen médical aux fins de l'immigration effectué au Canada, par année de test, 2009 à 2018

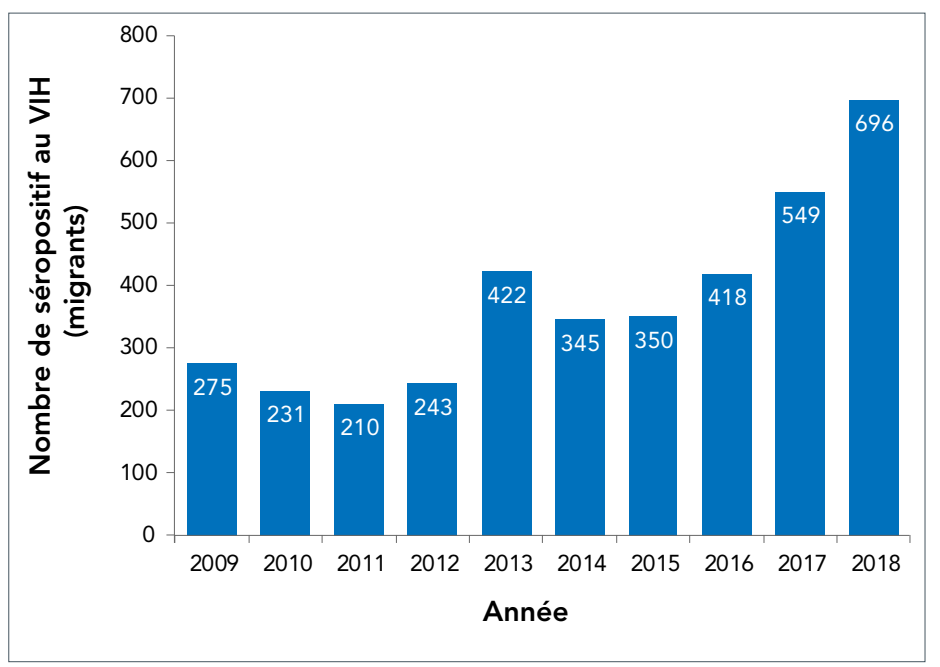

De 2009 à 2018, un total de 3739 candidats testés au Canada ont reçu un diagnostic de $\mathrm{VIH}$, avec une moyenne de 374 par année (étendue de 210 à 696). Un peu plus de la moitié des migrants séropositifs au VIH testés au Canada en 2018 étaient des hommes $(57,0 \%)$ et la majorité des candidats testés au Canada étaient âgés de 30 à 39 ans (39,8\%), suivis des 40 à 49 ans (26,4\%). Les groupes d'âge de 20 à 29 ans et 50 ans et plus représentaient $16 \%$ chacun du total. La majorité des candidats séropositifs au $\mathrm{VIH}$ résidaient en Ontario $(52,7 \%)$ et au Québec (29,3\%).

\section{Système de surveillance périnatale du VIH au Canada}

En 2018, au Canada, 259 nourrissons ont été exposés au VIH durant la période périnatale. De ce nombre, cinq nourrissons ont été confirmés séropositifs au VIH. Parmi ces cinq nourrissons, 
trois sont nés de mères qui n'ont pas reçu de traitement antirétroviral périnatal (TAR) prophylactique et deux sont nés de mères qui ont reçu un TAR prophylactique. Le nombre de nourrissons exposés durant la période périnatale et confirmés séropositifs au VIH a légèrement fluctué depuis 2011, mais est resté dans la fourchette de deux à neuf nourrissons séropositifs par an. Le pourcentage de mères séropositives recevant un traitement antirétroviral est demeuré relativement stable au cours des deux dernières années, soit 95,9\% en 2017 et $96,5 \%$ en 2018.

En 2018, l'exposition la plus fréquemment signalée chez les mères a continué d'être le contact hétérosexuel $(75,0 \%)$, suivi par l'utilisation de drogues par injection (20,1\%). En ce qui concerne la race ou l'origine ethnique des mères, en 2018, $54,4 \%$ des nourrissons exposés au VIH durant la période périnatale sont nés de mères identifiées comme noires. Viennent ensuite les nourrissons exposés au $\mathrm{VIH}$ nés de mères autochtones $(22,8 \%)$ et caucasiennes (16,2 \%). La région de naissance des mères de la majorité des nourrissons exposés au VIH durant la période périnatale était l'Amérique du Nord (45,2\%) et l'Afrique $(39,8 \%)$.

\section{Discussion}

Au Canada, 2561 diagnostics de VIH ont été signalés en 2018. Cela représente une augmentation de 8,2 \% par rapport à 2017 et une augmentation globale de $9,3 \%$ au cours de la dernière décennie. Le taux national de diagnostic est passé de 6,5 pour 100000 habitants en 2017 à 6,9 pour 100000 habitants en 2018. Il est difficile de comparer le taux de diagnostic du VIH au Canada de 2018 avec celui des autres pays à revenu élevé, car la plupart de ces pays n'ont pas encore publié leurs données de surveillance sur le VIH pour 2018. En ce qui concerne les données de 2017, les taux de nouveaux diagnostics de $\mathrm{VIH}$ variaient d'un pays à l'autre; par exemple, les États-Unis, I'Australie et le Royaume-Uni ont déclaré des taux nationaux de $11,8,4,0$ et 6,7 pour 100000 habitants, respectivement (17-19), par rapport au 6,5 pour 100000 habitants au Canada en 2017.

Comme nous l'avons déjà mentionné (2), I'augmentation du nombre de nouveaux diagnostics de VIH observée depuis 2014 au Canada peut être attribuable à plusieurs facteurs : une augmentation de la transmission du VIH (c.-à-d. I'incidence du VIH); une augmentation du nombre de test du VIH; des changements dans les pratiques de déclaration; et une augmentation du nombre de personnes séropositives migrant au Canada qui sont séropositives lors d'un premier test du VIH au Canada ou qui subissent un nouveau test au Canada (figure 6). Les données d'IRCC indiquent que, bien que la proportion de migrants séropositifs durant l'EMI soit demeurée relativement stable au cours des dernières années, le nombre total de personnes qui migrent au Canada a augmenté. Sur les 696 migrants qui se sont révélés séropositifs au VIH durant les
EMI au Canada en 2018, on ne sait pas la répartition exacte du nombre entre les personnes infectées à l'étranger et au Canada, puisque le délai entre l'arrivée au Canada et le moment où l'EMI est effectué varie. Cette distinction entre le VIH contracté à l'étranger et le VIH contracté au Canada est importante parce que la surveillance nationale porte sur l'épidémiologie de la transmission du VIH au Canada. Bien qu'un migrant infecté à l'étranger puisse être séropositif pour la première fois au Canada (et cette information est importante pour les mesures de la prévalence du $\mathrm{VIH}$ et la planification des services de santé), le fait de considérer le cas comme un "nouveau » diagnostic complique l'interprétation et l'utilisation du taux national de diagnostic comme indicateur de la transmission au Canada. D'autres organismes, comme le Centre européen de prévention et de contrôle des maladies, élaborent des méthodes pour faire la distinction entre ces deux groupes, et l'ASPC espère s'inspirer des conversations avec IRCC pour faire de même.

Une première étape pour identifier les migrants infectés par le VIH à l'extérieur du Canada (et les distinguer des dénombrements nationaux de surveillance du VIH) consiste à identifier les personnes qui déclarent une séropositivité antérieure au $\mathrm{VIH}$ dans un autre pays. L'ASPC a récemment analysé les données nationales entre 2007 et 2017 afin d'identifier les cas ayant une définition commune de " séropositivité antérieure au VIH » pour quantifier l'effet de tous les cas déjà diagnostiqués, dans un autre pays ou une autre province, sur la tendance nationale. Le pourcentage d'augmentation des diagnostics de $\mathrm{VIH}$ au cours de cette période est passé de $16 \%$ (signalé dans le précédent rapport annuel de surveillance) (2) à $9 \%$ lorsque les cas précédemment diagnostiqués ont été supprimés (10). À l'avenir, les rapports nationaux de surveillance présenteront les tendances des nouveaux diagnostics de $\mathrm{VIH}$ (aux pour lesquels on ne connait pas l'existence d'un diagnostic antérieur) séparément de tous les diagnostics de VIH. La poursuite de ces travaux permettra de préciser le nombre et le taux des nouveaux diagnostics, ce qui résultera dans l'obtention de renseignements plus précis sur la transmission du VIH au Canada.

Comme par le passé, la catégorie d'exposition des gbHARSAH inclut la plus grande proportion de nouveaux diagnostics de VIH et représentait plus de la moitié des cas chez les hommes adultes $(58,1 \%)$ en 2018 . Toutefois, la proportion de cas de gbHARSAH a diminué au fil des ans. Par ailleurs, depuis 1996, le taux de diagnostics de $\mathrm{VIH}$ chez les femmes a fluctué entre 2,5 et 4,2 pour 100000 habitants. Au cours des cinq dernières années, le taux est passé de 2,5 pour 100000 habitants à 4,0 pour 100000 habitants en 2018. Les provinces de la Colombie-Britannique, de la Saskatchewan, du Manitoba, de I'Ontario et du Québec ont observé une croissance des taux chez les femmes en 2018 par rapport à 2017. On n'a pas observé d'augmentation des taux chez les femmes dans d'autres pays à revenu élevé : les taux de diagnostic chez les femmes en Australie ont été stables, les taux les plus élevés étant chez les femmes âgées de 30 à 39 
ans (19), tandis qu'on a signalé une baisse des taux de diagnostic chez les femmes aux États-Unis et au Royaume-Uni $(17,18)$.

Cette augmentation chez les femmes au Canada est observée dans toutes les catégories d'exposition et les données futures aideront à déterminer son importance.

\section{Points forts et limites}

La force principale de ce rapport repose dans le fait qu'il s'agit de la seule source de données épidémiologiques nationales sur tous les diagnostics déclarés de VIH au Canada.

Les limites du SSVS ont été décrites précédemment $(2,14)$. L'une des limites à noter est la variation dans la déclaration des cas déjà diagnostiqués entre les provinces et les territoires; l'inclusion de certains de ces cas a probablement entraîné un sur dénombrement des nouveaux cas de VIH au Canada (10). L'information future sur la surveillance comprendra des méthodes pour atténuer cette limitation. De plus, il n'a pas été possible de tirer des conclusions sur la race ou l'origine ethnique des nouveaux diagnostics de VIH signalés en 2018 en raison de données manquantes dans les déclarations provinciales et territoriales. À l'avenir, I'ASPC collaborera avec ses partenaires de surveillance pour améliorer l'information sur la race et l'origine ethnique afin de comprendre ces tendances chez les nouveaux cas de diagnostic de VIH.

Les données contenues dans le présent rapport sont considérées comme provisoires et peuvent faire l'objet de modifications dans les futurs rapports de surveillance du VIH. S'il y a des écarts entre les données résumées dans ce rapport et les rapports provinciaux et territoriaux, le plus récent rapport provincial et territorial devrait être utilisé parce que les données nationales risquent de ne pas être encore à jour.

\section{Conclusion}

Le nombre et le taux de cas déclarés de VIH au Canada ont augmenté en 2018, ce qui pourrait être attribuable en partie aux cas diagnostiqués pour la première fois dans une province ou un territoire, mais qui avaient déjà été diagnostiqués soit dans d'autres provinces ou à l'étranger. La catégorie d'exposition des gbHARSAH a continué de représenter la plus forte proportion de la catégorie d'exposition au VIH au Canada, bien que cette proportion ait diminué. Le nombre et le taux de nouveaux diagnostics ont augmenté chez les femmes de toutes les catégories d'exposition. L'ASPC continuera de collaborer avec les provinces et les territoires pour décrire plus précisément les tendances de la transmission du VIH au Canada, y compris l'identification des cas déjà diagnostiqués.

\section{Déclaration des auteurs}

N. H. - Conceptualisation, recherche, rédaction, ébauche initiale, ébauche finale, révision, correction, validation de données, visualisation, supervision

A. R. - Gestion de données, validation de données, recherche
A. W. - Validation de données, correction, recherche

N. P. - Révision, correction, supervision

W. S. - Révision, correction, supervision

C. A. - Révision, correction

\section{Conflit d'intérêts}

Aucun.

\section{Remerciements}

L'Agence de la santé publique du Canada aimerait remercier les employés suivants des programmes provinciaux et territoriaux de lutte contre le $\mathrm{VIH}$ et le SIDA pour leur contribution et leur participation: S. Fathima, ministère de la Santé de l'Alberta; A. O'Brien, ministère de la Santé de l'Alberta; J. Wong, Service de prévention clinique, Surveillance et épidémiologie, Centre for Disease Control de la Colombie-Britannique; C. Loeppky, ministère de la Santé du Manitoba; R. RaaFat Gad, ministère de la Santé du Nouveau-Brunswick; S. Landsburg, ministère de la Santé du Nouveau-Brunswick; S. Wertz, ministère de la Santé du Nouveau-Brunswick; B. Halfyard, Santé et services communautaires, Direction générale de la santé de la population, Terre-Neuve-et-Labrador; M. O'Driscoll, Santé et services communautaires, Direction générale de la santé de la population, Terre-Neuve-et-Labrador; H. Hannah, ministère de la Santé et des Services sociaux, Territoires du Nord-Ouest; B. Billard, ministère de la Santé et du Mieux-être de la Nouvelle-Écosse; S. Fleming, ministère de la Santé et du Mieux-être de la Nouvelle-Écosse; K. Kullerperuma, ministère de la Santé du Nunavut; $A$. Kroch, Réseau ontarien de traitement du VIH; J. Liu, Santé publique Ontario; M. Murti, Santé publique Ontario; M. Whelan, Santé publique Ontario; S. Baidoobonso, Santé et Mieux-être, île-du-Prince-Édouard; C. Cheverie, Santé et Mieux-être, Île-du-Prince-Édouard; R. Bitera, Direction des risques biologiques et de la santé au travail, Institut national de santé publique du Québec; M. Fauvel, Laboratoire de santé publique du Québec, Institut national de santé publique du Québec; M. Hastie, Laboratoire de santé publique du Québec, Institut national de santé publique du Québec; R. Parent, Direction des risques biologiques et de la santé au travail, Institut national de santé publique du Québec; B. Serhir, Laboratoire de santé publique du Québec, Institut national de santé publique du Québec; D. Sylvain, Laboratoire de santé publique du Québec, Institut national de santé publique du Québec; H. Bangura, ministère de la Santé de la Saskatchewan; J. Manalo, ministère de la Santé de la Saskatchewan; R. Wallace, Contrôle des maladies transmissibles Yukon; L. Strudwick, Contrôle des maladies transmissibles Yukon; S. Plitt, Agence de la santé publique du Canada, Alberta; E. Wong, Agence de la santé publique du Canada, Colombie-Britannique; J. Paul, Public Agence de la santé publique du Canada, Manitoba; H. Rilkoff, Agence de la santé publique du Canada, Ontario; T. Schellenberg, Agence de la santé publique du Canada, 
Saskatchewan; M. Nichols Agence de la santé publique du Canada, Nouvelle-Écosse.

\section{Financement}

Cet article a reçu l'appui financier de l'Agence de la santé publique du Canada dans le cadre de son mandat de base.

\section{Références}

1. The Joint United Nations Programmed on HIV/AIDS. UNAIDS Data 2018. UNAIDS: 2018. https://www.unaids.org/en/ resources/documents/2018/unaids-data-2018

2. Haddad N, Li JS, Totten S, McGuire M. Le VIH au Canada, Rapport de surveillance, 2017. Relevé des maladies transmissibles au Canada 2018;44(12):367-76. DOI

3. Global Burden of Disease Health Financing Collaborator Network. Spending on health and HIV/AIDS: domestic health spending and development assistance in 188 countries, 19952015. Lancet 2018 May;391(10132):1799-829. DOI PubMed

4. Agence de la santé publique du Canada. Réduction des répercussions sur la santé des infections transmissibles sexuellement et par le sang au Canada d'ici 2030 : un cadre d'action pancanadien sur les ITSS Ottawa (ON); ASPC : 2019. https://www.canada.ca/fr/sante-publique/services/ maladies-infectieuses/sante-sexuelle-infections-transmissiblessexuellement/rapports-publications/infections-transmissible s-sexuellement-sang-cadre-action.html

5. Agence de la santé publique du Canada. Accélérer notre intervention : plan d'action quinquennal du gouvernement du Canada sur les infections transmissibles sexuellement et par le sang. Ottawa (ON); ASPC : 2019. https://www.canada.ca/fr/ sante-publique/services/rapports-publications/accelerer-notr e-intervention-plan-action-quinquennal-infections-transmissibles -sexuellement-sang.html

6. Cohen MS, Chen YQ, McCauley M, Gamble T, Hosseinipour MC, Kumarasamy N, Hakim JG, Kumwenda J, Grinsztejn B, Pilotto JH, Godbole SV, Mehendale S, Chariyalertsak S, Santos BR, Mayer KH, Hoffman IF, Eshleman SH, Piwowar-Manning E, Wang L, Makhema J, Mills LA, de Bruyn G, Sanne I, Eron J, Gallant J, Havlir D, Swindells S, Ribaudo H, Elharrar V, Burns D, Taha TE, Nielsen-Saines K, Celentano D, Essex M, Fleming TR; HPTN 052 Study Team. Prevention of HIV-1 infection with early antiretroviral therapy. N Engl J Med 2011 Aug;365(6):493-505. DOI PubMed

7. Marks G, Crepaz N, Senterfitt JW, Janssen RS. Meta-analysis of high-risk sexual behavior in persons aware and unaware they are infected with HIV in the United States: implications for HIV prevention programs. J Acquir Immune Defic Syndr 2005 Aug;39(4):446-53. DOI PubMed
8. Metsch LR, Pereyra M, Messinger S, Del Rio C, Strathdee SA, Anderson-Mahoney P, Rudy E, Marks G, Gardner L; Antiretroviral Treatment and Access Study (ARTAS) Study Group. HIV transmission risk behaviors among HIV-infected persons who are successfully linked to care. Clin Infect Dis 2008 Aug;47(4):57784. DOI PubMed

9. Antiretroviral Therapy Cohort Collaboration. Life expectancy of individuals on combination antiretroviral therapy in high-income countries: a collaborative analysis of 14 cohort studies. Lancet 2008 Jul;372(9635):293-9. DOI PubMed

10. Popovic N, Yang Q, Haddad N, Weeks A, Archibald C. Améliorer la surveillance nationale des nouveaux diagnostics du $\mathrm{VIH}$. Relevé des maladies transmissibles au Canada 2019;45(12):3447. DOI

11. Agence de la santé publique du Canada. Système canadien de surveillance des maladies à déclaration obligatoire. Maladies à déclaration obligatoire en direct. Ottawa (ON); ASPC : 2019. https://maladies.canada.ca/declaration-obligatoire/

12. Agence de la santé publique du Canada. Résumé : Estimations de l'incidence et de la prévalence du VIH, et des progrès réalisés par le Canada en ce qui concerne les cibles 90-90-90 pour le VIH, 2016. Ottawa (ON); ASPC : 2018. https://www. canada.ca/fr/sante-publique/services/publications/maladies-etaffections/esume-estimations-incidence-prevalence-vih-progr es-realises-canada-90-90-90.html

13. Agence de la santé publique du Canada. Définitions nosologiques des maladies transmissibles faisant l'objet d'une surveillance nationale - 2009. Relevé des maladies transmissibles au Canada 2009;35(S2):S1-123. https://www.canada.ca/ content/dam/phac-aspc/migration/phac-aspc/publicat/ ccdr-rmtc/09pdf/35s2-fra.pdf

14. Agence de la santé publique du Canada. Le VIH et le sida au Canada - Rapport de surveillance en date du 31 décembre 2014. Ottawa (ON); ASPC : 2015. https://www.canada.ca/fr/ sante-publique/services/publications/maladies-et-affections/ vih-et-sida-canada-rapport-surveillance-31-decembre-2014.html

15. Statistique Canada. Estimations démographiques annuelles: Canada, provinces et territoires. 2017. Ottawa (ON): StatsCan; 2018. https://www150.statcan.gc.ca/n1/fr/ catalogue/91-215-X

16. Statistique Canada. Guide de référence sur l'origine ethnique, Recensement de la population, 2016. Ottawa (ON): StatsCan; 2017. https://www12.statcan.gc.ca/census-recensement/2016/ ref/guides/008/98-500-x2016008-fra.cfm

17. Centers for Disease Control and Prevention. HIV Surveillance Report, Volume 29: Diagnoses of HIV Infection in the United States and Dependent Areas, 2017. CDC: 2018. https://www.cdc.gov/hiv/pdf/library/reports/surveillance/ cdc-hiv-surveillance-report-2017-vol-29.pdf

18. Public Health England. Progress towards ending the HIV epidemic in the United Kingdom - 2018 report. London (UK); 2018. https://assets.publishing.service.gov.uk/government/ uploads/system/uploads/attachment_data/file/821273/Progress_ towards_ending_the_HIV_epidemic_in_the_UK.pdf

19. University New South Wales. Australia. The Kirby Institute. HIV, viral hepatitis and sexually transmissible infections in Australia: Annual surveillance report 2018. Sydney, Australia; UNSW; 2018. https://kirby.unsw.edu.au/report/hiv-viral-hepatitis-and-sexual ly-transmissible-infections-australia-annual-surveillanceAnnexe : Liste des tableaux supplémentaires 


\section{Annexe : Liste des tableaux supplémentaires Ces tableaux sont disponibles sur demande à phac.hass.aspc@canada.ca.}

Tableau S1 : Taux de diagnostics du VIH (pour 100000 habitants) par province ou territoire et année de diagnostic (tous les âges)

Tableau S2: Taux de cas de VIH (tous les âges) par province ou territoire, sexe et année du diagnostic - Canada, 1985 à 2018

Tableau S3 : Taux de cas de VIH par groupe d'âge et province ou territoire - Canada, 2017 à 2018

Tableau S4 : Nombre cumulatif de cas de VIH chez les adultes (15 ans ou plus) et les enfants (moins de 15 ans) par sexe - Canada, du $1^{\text {er }}$ novembre 1985 au 31 décembre 2018

Tableau S5 : Nombre de cas de VIH chez les adultes (15 ans ou plus) par année de diagnostic et par sexe - Canada, 1985 à 2018

Tableau S6 : Nombre de cas de VIH par groupe d'âge, sexe et année du diagnostic - Canada, 1985 à 2018

Tableau S7 : Nombre et répartition en pourcentage de cas de VIH chez les adultes (15 ans ou plus) par catégorie d'exposition et année du diagnostic - Canada, 1985 à 2018

Tableau S8 : Nombre et répartition en pourcentage de cas de $\mathrm{VIH}$ chez les hommes adultes (15 ans ou plus) par catégorie d'exposition et année du diagnostic - Canada, 1985 à 2018

Tableau S9 : Nombre et répartition en pourcentage de cas de $\mathrm{VIH}$ chez les femmes adultes (15 ans ou plus) par catégorie d'exposition et année du diagnostic - Canada, 1985 à 2018

Tableau S10 : Nombre et répartition en pourcentage de cas de VIH chez les adultes (15 ans ou plus) par catégorie d'exposition et groupe d'âge - Canada, 2016 à 2018

Tableau S11 : Nombre de cas de VIH par catégorie d'exposition et province ou territoire - Canada, 2016 à 2017
Tableau S12 : Nombre et répartition en pourcentage de candidats à l'immigration au Canada diagnostiqués par année à la suite d'un examen médical aux fins de l'immigration 2002 à 2018

Tableau S13 : Nombre et répartition en pourcentage de candidats à l'immigration au Canada diagnostiqués à la suite d'un examen médical aux fins de l'immigration par sexe, groupe d'âge et province - 2002 à 2018

Tableau S14 : Nombre de nourrissons exposés au VIH durant la période périnatale par année de naissance, statut actuel et utilisation de la thérapie antirétrovirale pour la prophylaxie Canada, 1984 à 2018

Tableau S15 : Nombre de nourrissons exposés au VIH durant la période périnatale par catégorie d'exposition maternelle et année de naissance - Canada, 1984 à 2018

Tableau S16 : Nombre de nourrissons exposés au VIH durant la période périnatale par origine ethnique et statut d'infection Canada, 1984 à 2018

Tableau S17 : Nombre de nourrissons exposés au VIH durant la période périnatale par pays d'origine maternel et statut d'infection - Canada, 1984 à 2018

Tableau S18 : Nombre de nourrissons exposés au VIH durant la période périnatale par région géographique et statut au dernier rapport - Canada, 1984 à 2018

Tableau S19 : Statistiques internationales sur les cas de VIH signalés - Canada, 2017

Tableau S20 : Taux de cas de VIH par groupe d'âge, sexe et année du diagnostic - Canada, 2014 à 2018 